Original Research Paper

\title{
Biofidel FEA Modeling of Customized Hybrid Biological Hip Joint Prostheses, Part I: Biomechanical Behavior of Implanted Femur
}

\author{
${ }^{1}$ Raffaella Aversa, ${ }^{2}$ Florian Ion T. Petrescu, ${ }^{3}$ Relly Victoria V. Petrescu and ${ }^{1}$ Antonio Apicella \\ ${ }^{I}$ Advanced Material Lab, Department of Architecture and Industrial Design, Second University of Naples, Aversa (CE) Italy \\ ${ }^{2}$ Department of Theory of Mechanisms and Robots, Bucharest Polytechnic University, Bucharest, Romania \\ ${ }^{3}$ Department of Transport, Traffic and Logistics, Bucharest Polytechnic University, Bucharest, Romania
}

Article history

Received: 07-10-2016

Revised: $15-12-2016$

Accepted: 16-12-2016

Corresponding Author: Florian Ion T. Petrescu Department of Theory of Mechanisms and Robots, Bucharest Polytechnic University, Bucharest, Romania Email: scipub02@gmail.com

\begin{abstract}
Biofidel femur Finite Element Models have been developed using specific combination of Computer Tomography segmentation and solid modeling software tools able to represent bone physiology and structural behavior. These biofidel Finite Element Models (FEM) is used to evaluate the modification of the physiological stress distribution in a prosthesized femur and to assess new design criteria for the development of biomimetic hybrid biological hip prostheses. The faithful models proposed allowed us to properly consider the not isotropic characteristics of the proximal epiphysis of the femur and for the isotropic behavior in diaphysis to explain the critical alterations of the stress distribution in a resected femur following the implantation of a traditional hip joint prostheses. It has been shown that a wide region of the femur diaphysis is completely shielded by the rigid prosthesis significantly altering the physiological stress distribution that should guaranty a healthy bone growth and regeneration.
\end{abstract}

Keywords: Trabecular Bone, Biomimetic, Biomechanics, Trabecular Prostheses

\section{Introduction}

A highly interdisciplinary research group has been involved in the study of bone as a living material. The principal efforts have been devoted to mimic the bone characteristics and process of its formations and behavior under physiological loading (Apicella et al., 2010; 2011; 2015; Gramanzini et al., 2016; Kummer, 1986; Perillo et al., 2010; Sorrentino et al., 2009; 2007).

Advanced academic studies on biomechanics and biomimetic of implanted bones resulted from these investigations. This work project identifies a number of design criteria to foster their potential to enable new medical therapies contributing to customized health care and to create and improve the technological basis for innovative prostheses design.

The human femur is characterized by a specific internal structure (Oh and Harris, 1976; Gottesman and Hashin, 1980) that imparts to the bone a high capacity to withstand external stresses while optimizing its mass distribution and morphology (Ashman et al., 1984; Dalstyra et al., 1993). However, physiological bone mass loss progressively occurs at older age, reducing the its toughness and capability to dissipate the energy transmitted by external shock event and this feature is the cause of most of the elder people pertrochanteric fractures along the plane indicated in Fig. 1.

Aversa et al. (2016) have successfully modeled this behavior. Pertrochanteric fractures need hip joint prosthesis application (Ashman and Rho, 1988; Burnstein et al., 1976; Carter and Hayes, 1977). The modification of the biomechanical behavior of aged or implanted bones can be correctly predicted using our previously developed biofidel Finite Element Modeling (FEM) (Apicella et al., 2010; Aversa et al., 2009).

Nonetheless, total hip replacements performed on patients younger than 65 and hence with a necessary longer expected durability of the orthopedic implant, cannot endure for more than 15 years. Nevertheless, this clinical scenario has been changing now. Several health care technological developments increased the life expectations. Moreover, the prognosis for physical trauma due to sports, improper and excessive exercise practice, or road crashes have been ameliorated. New design more long lasting and biomechanically compatible prostheses are then needed. 


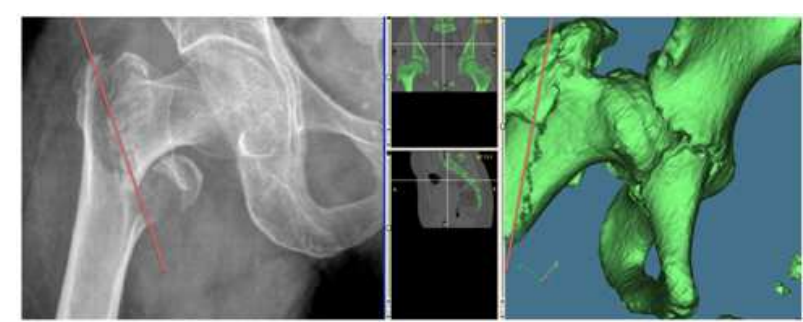

Fig. 1. Fracture planes (red line) of a pertrochanteric femur fracture (left CT) and solid modeling from software for $\mathrm{CT}$ segmentation in the proximal epiphysis-pelvis region

The orthopedic prostheses used today are made of metal alloys, plastics and ceramics materials with well defined properties and characteristics. In particular, due to their high biocompatibility and high mechanical strength, the metal alloys commonly used are based on Titanium or Cobalt Chrome and they represent almost all of the prosthetic components that are in direct contact with the human bone since they can provide a good osteo-integration with the bone components.

Implant failures are principally due to biomechanical incompatibility between the prosthesis and the bone, namely, the inadequate strength or mismatching of the rigidities between the bone and implant causes arise of discontinuities in the stress and strains distribution in the bone with respect to the biologically one. The current implant materials (metal and ceramic), which are more stiffer than bone, strongly alters the physiological bone stress distribution and prevents the needed stress being transferred to adjacent bone (Apicella et al., 2010; 2011; 2015), resulting in bone reabsorption near the implant and, thus, increasing the risk of implant loosening.

In order to predict the structural alterations induced by the modification of the morphological and mechanical characteristic of the bone, faithful models of the femur have been developed (Aversa et al., 2016). The development of these bio-models has already enabled us to correctly predict physiological stress and strain distribution in the mandible and dental prostheses implant (Apicella et al., 2010; Gramanzini et al., 2016; Perillo et al., 2010; Sorrentino et al., 2009; 2007).

In the present work a femur FEM model, which has been developed in a previous work to correctly represent structural behavior of the femur head (Aversa et al., 2016) has been analyzed for the stress and strains distribution over the entire bone stem and head and it has been modified to account for femur head resection and substitution with a Titanium hip joint prosthesis in the case of a pertrochanteric femur fracture (Fig. 1). A comparison between the biological stress and strain distributions in the femur models of the sound and prothesized femurs could help in the understanding of the correct design procedures needed to design new innovative biomimetic prostheses.

\section{Materials and Methods}

Image Segmentation derived from CT has been performed using the Mimics software (Materialise, Belgium) to process a patient tomography. An accurate 3D solid model of the patient pelvis and femur anatomy, Fig. 2, has been derived by processing of CT data.

New prostheses design through a combined use of Mimics and 3-Matic (Materialise, Belgium) software's could be derived from the study of the biomechanics of these bone districts. Combined solid modeling and Finite Element analysis have been developed in a previous work by Aversa et al. (2016). This methods simulates the structural morphology of the femur as it was already made for other complex bone structures that consider orientation and densities of the trabecular bone (Apicella et al., 2010; 2011; 2015; Aversa et al., 2016; 2009; Beaupre and Hayes, 1985; Reilly and Burstein, 1974; 1975; Huiskes et al., 1987; Taylor et al., 2007; Rohlmann et al., 1982). Several recent investigations underlined the importance of the FEM analysis in clinical applications and for the development of new prosthetic systems (Mirsayar and Park, 2016; Mazaheri et al., 2016) and for the use of innovative materials and surface treatments (Kumar et al., 2016).

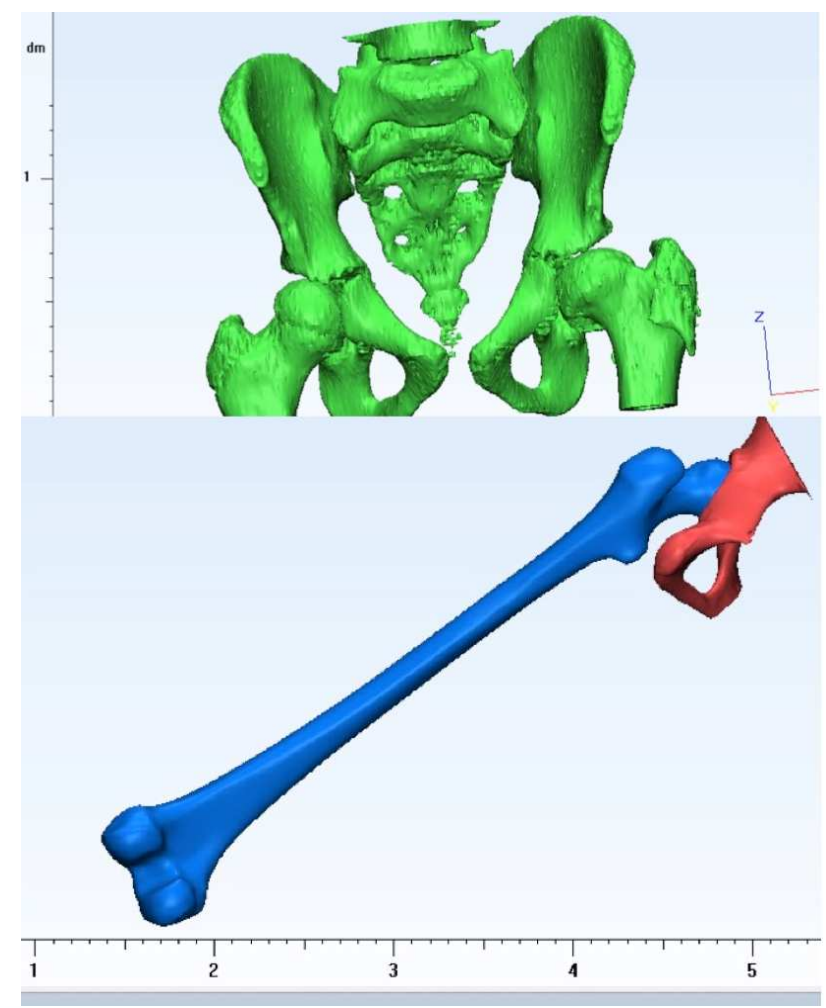

Fig. 2. Biofidel medical Image Segmentation of a patient pelvis and femur 


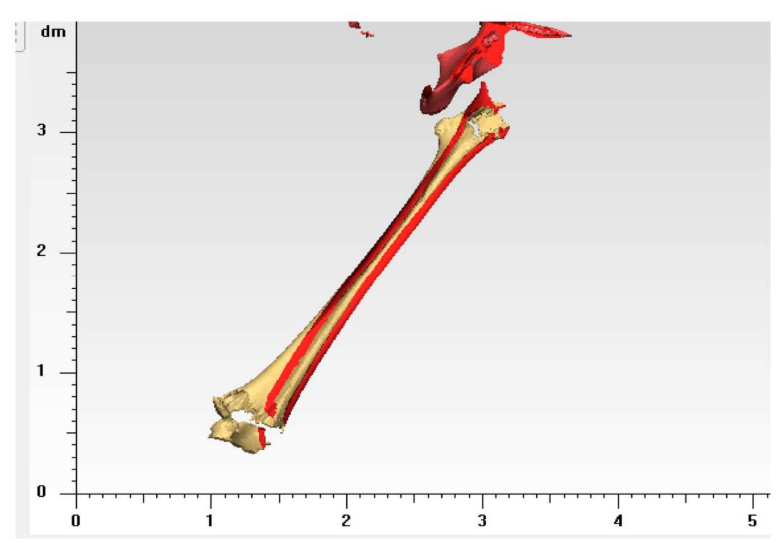

Fig. 3. Biofidel 3D solid modelling of a patient femur and pelvis
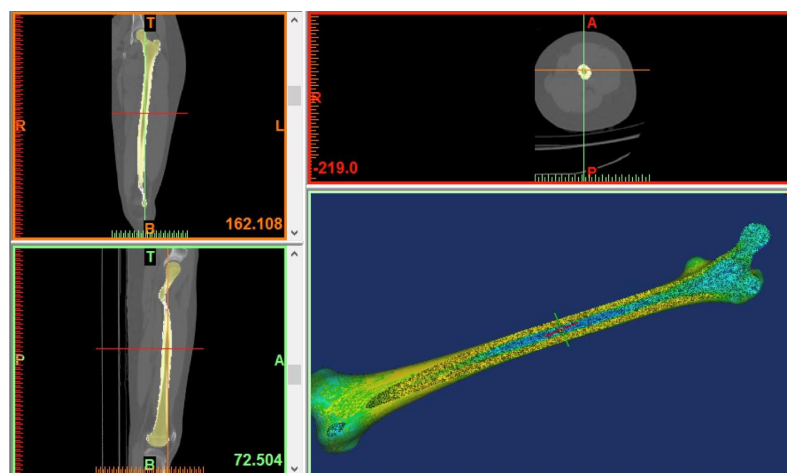

Fig. 4. Mesh creation and optimization of the biofidel not resected femur model (Aversa et al., 2016)

The methodological procedure is illustrated in the Fig. 3-6. The external geometry of femur and portion of the pelvis have been remodeled creating a $3 \mathrm{D}$ volume from the CT scans (Fig. 3).

The models were imported in the 3Matic software in order to create and optimize surface and solid meshing, which was needed to the preparation of the Finite element model and for the designation of material properties (Aversa et al., 2016).

The results of tethraedric element material distribution are shown in Fig. 4.

The same procedure has been applied to the preparation of the FEA model of the femur with the resection of the head (Fig. 5), which has been virtually related to the fracture plane reported in Fig. 1 and for the Titanium traditional hip-joint prosthesis (reported in the Left side of Fig. 6).

The model of the resected femur and of the Titanium prosthesis has been realized by definition of the $3 \mathrm{D}$ tethraedric mesh optimization of the distribution and size, as indicated in the right side of Fig. 5 and 6, respectively.

The solid mesh elements of the resected femur have been associated to the bone densities such as measured by a Hounsfield (HU) scale, which compute the X-rays linear attenuation coefficients of in the tissues, by the Mimics software (lower part of Fig. 5).
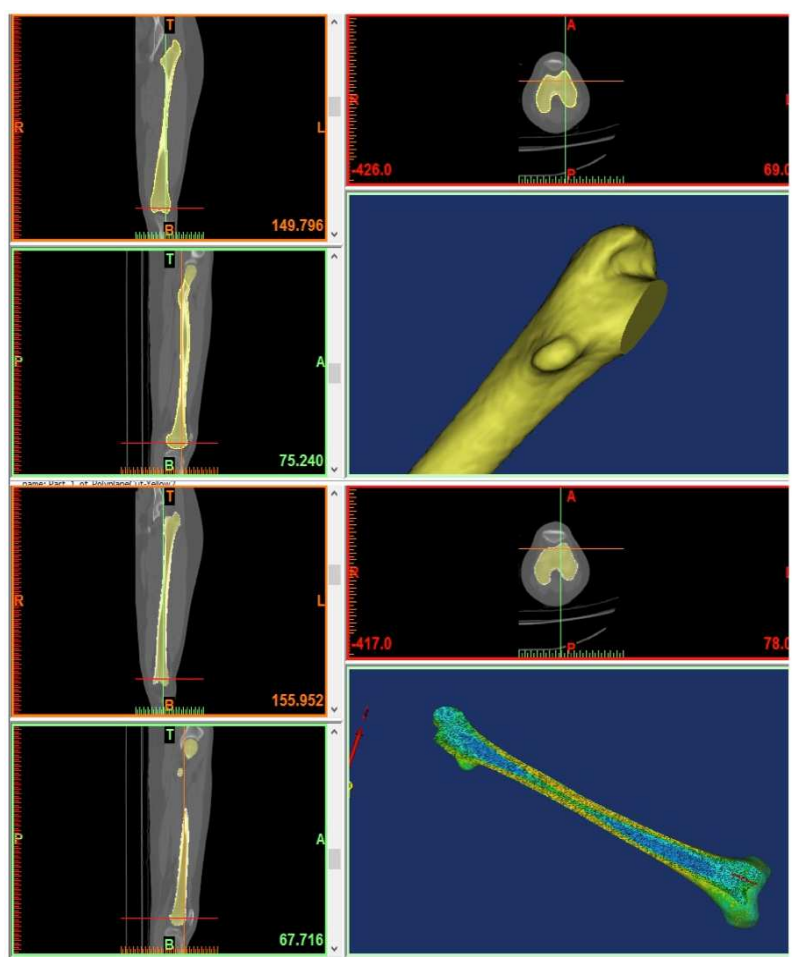

Fig. 5. 3D meshing optimization of the biofidel patient femur model
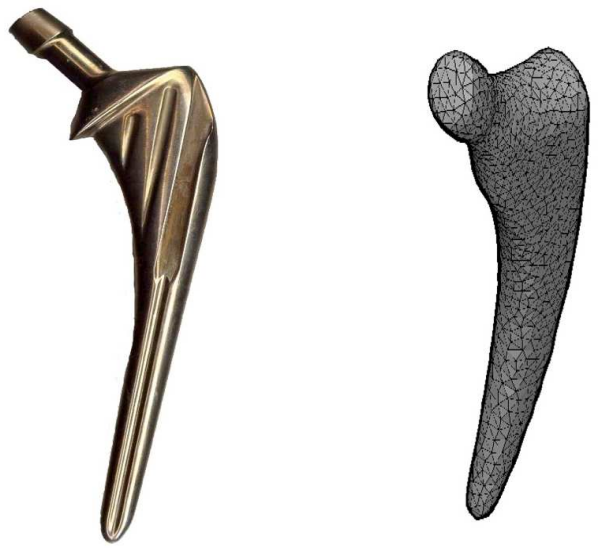

Fig. 6. 3D meshing optimization of a Titanium traditional prosthesis

The meshed solid models of the resected femur and of the Ti prosthesis were assembled accounting to the correct positioning of the implant using the Mimics software (Fig. 7). New remeshing of the tethraedric elements were run in 3Matic (Fig. 8) searching for the node congruency at the bone-implant interfaces, where the material definition has been made for the new finite elements configuration. The evaluation of mechanical properties has been done considering the cortical and trabecular bone mechanical characteristics. 


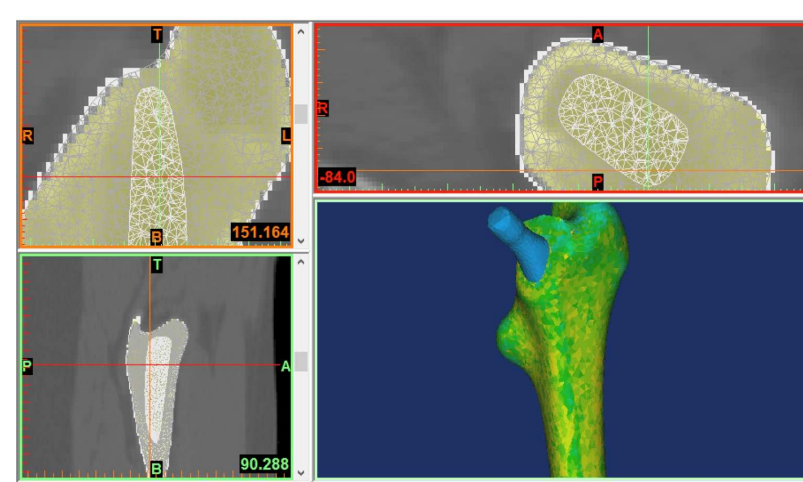

Fig. 7. Assembly and material properties definition associated to the patient femur cortical and trabecular bone densities and to the Titanium prosthesis

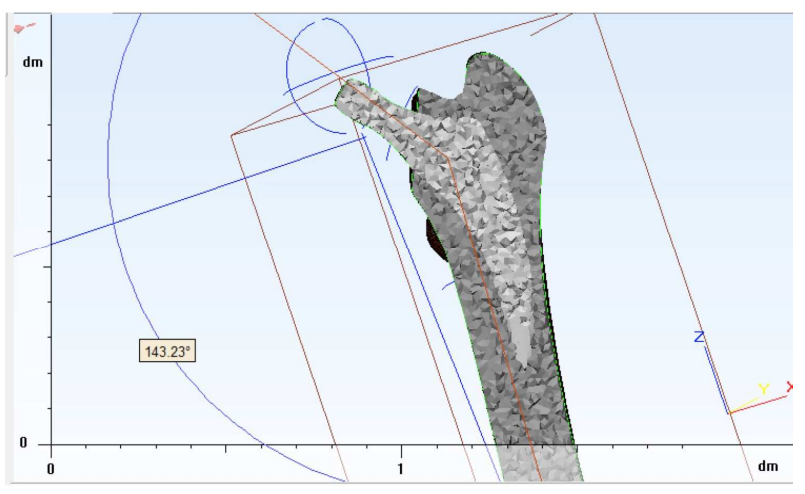

Fig. 8. Assembly of the resected femur and Titanium rigid hip prosthesis and tethraedric mesh congruency verification

In the Hounsfield (HU) scale, the trabecular bone is in the range 100-300, while the cortical bone covers values from 200 up to about 2000, which corresponded in our bone systems to Elastic moduli ranging from 0,87 to $15.0 \mathrm{GPa}$. The fat value is about -110 , the muscle about 40. Finally, the mechanical properties of the prosthesis Ti alloy are: Elastic modulus equals to 124 $\mathrm{GPa}$ and Poisson ratio 0.3.

\section{FEM Analysis: Sound and Prosthesis Femur}

On the basis of the 3D models of the sound femur and the resected femurs implanted with a traditional rigid prosthesis, a structural evaluation under the same loading conditions has been developed and the results critically compared.

The personal characteristic biometric parameters of the patient femur-hip system have been carried out before running the structural analysis.

The 3Matic software has been used to identify the direction of the loading axis, the center of the proximal epiphysis head sphere and the center of the joint epicondyle and mechanical axis of rotation of the knee.

Namely, the following parameters have been measured: The mechanical axis between the centers of the proximal and distal epiphyses, the angle of $143.40^{\circ}$ between the femoral neck and diaphysis axes and the divergence of $36.65^{\circ}$ between the neck axis with the axis of epicondyles.

Finally (before running the FEM structural analyses) the physiological loads and constrains have been evaluated.

As limiting bone load condition, the equilibrium monopodalic posture has been chosen where the gluteus muscular force balances the moment of body weight force. For a body weight of $100 \mathrm{Kg}$ and accordingly to the previously defined biometric parameters, the gluteus muscular force applied to the great trochanter is about $1800 \mathrm{~N}$ and the reaction force at the joint $2740 \mathrm{~N}$. (Kumar et al., 2016; Annunziata et al., 2006).

The equilibrium condition and femur-loading scheme are illustrated in Fig. 9.

Reaction forces generated by the gluteus have been uniformly distributed over 100 nodes of great trochanter surface. The reaction forces acting at level of the femur joint are distributed on 50 nodes of the femur head (as indicated in the upper left of Fig. 9).

\section{Results and Discussion}

This study defines a biofidel model to investigate the femur structural behavior. Von Mises strain criterion has been used to compare the stress distributions in sound and prosthesis femur under the previously defined loading conditions.

This Von Mises is an energetic criterion that can better quantify the capability of the sound femur to withstand high loads (right part of Fig. 9).

Posteriorly, stress distribution confirms the presence of bending effects, with the highest values homogeneously distributed in the anatomical diaphysis regions (right hand side of Fig. 9) with a maximum of about $90 \mathrm{MPa}$.

The same structural analysis has been carried out on the on the FE model of the prosthesized femur described in Fig. 7 and 8. The results of the analysis are compared in Fig. 10 to those evaluated for the sound femur.

Figure 10 shows the significant alteration of the stress distribution in the sound femur (left hand side) and the resected femur with the rigid $\mathrm{Ti}$ prosthesis (right hand side). Compared to the physiological flexural stresses in the diaphysis of the sound femur, the prosthesized femur induces a concentration in the medial region while they are completely absent in the proximal end. This occurrence is referred as "stress shielding effect" and it is due to the high rigidity of the metal prosthesis stem that does not allow the physiological flexure of diaphysis.

The absence of stress (and related strains state) could induce, over the time, significant bone structure modification (bone reabsorption). 


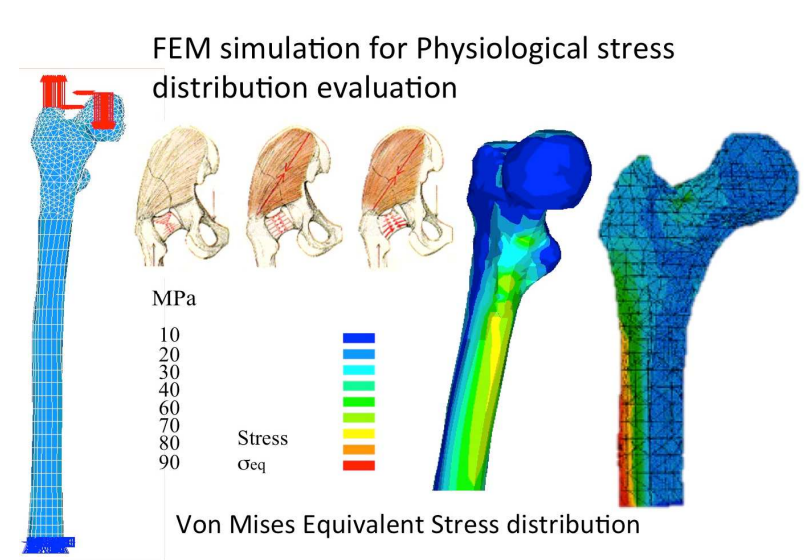

Fig. 9. Phisiological Equivalent Von Mises stresses in the femur from our biofidel Finite Element Model

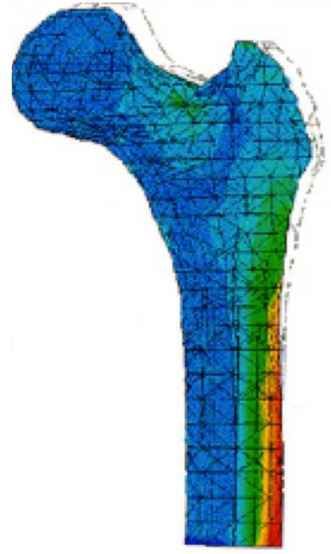

(a)

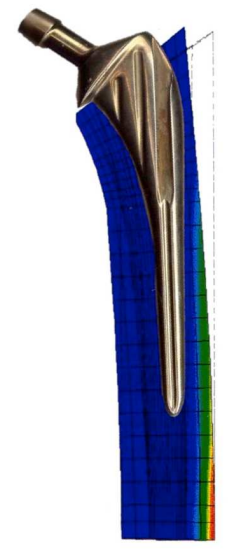

(b)
Fig. 10. Equivalent Von Mises stresses distribution in the proximal femur epiphysis for (a) sound femur, (b) rigid Ti prosthesis

Bone modeling and remodeling processes, in fact, enable itself to grow, renew and repair itself (Gottesman and Hashin, 1980; Huiskes et al., 1987; Taylor et al., 2007; Weinans et al., 1992). Mechanical adaptation of trabecular density and architecture could be explained in terms of mechano-sensory and signaling functions for the osteocytes (Mullender and Huiskes, 1995). Mechanic-regulation mechanism driving bone remodeling uses mechanical strain and micro-damage as the stimulus driving cellular responses and the consequent changes in the bone density and geometry. The absence or the modification of the biomechanical stimuli shown in Fig. 10 for the femur diaphysis after prosthesis implantation is then expected to lead, over the time, to a bone remodeling and reabsorption due to the absence of an adequate stress and strain state.

\section{Conclusion}

Results of our biofidel modeling of the sound and prosthesized bone would clarify the level of complexity needed when computing the strain state of a human femur through FEA when the stress and strain state has to be used to quantify the bone remodeling process.

A faithful bone models allows the accurate choice of the femur locations where the hypothesis of isotropic conditions (diaphysis) can be made and the femur locations where the bone orthotropy hypothesis should be applied (proximal end epiphysis). The distribution of maximum strain is correctly described by our theoretical approach since can envisage the location of the pertrochanteric fracture. The model can be usefully applied in those cases where the strain distributions need to be evaluated in order to predict undesired bone remodeling processes.

A further field of application of the product is surgical new prostheses design for orthopedic oncology to support and facilitate bone regeneration resulting in massive losses due to interventions of removal of both primitive and metastatic tumors.

The prosthetic system could be redesigned to a better functional recovery by promoting bone recreation by bone growth stimulation while ensuring a good hold of the load.

These improvements of the system biomimetic characteristics positively impact on the quality of life of the individual patient, which was already seriously compromised by the basic oncological pathology.

\section{Acknowledgement}

We acknowledge Taher M. Abu-Lebdeh, Assoc. Pro. At North Carolina A and T State Univesity, United States and Shweta Agarwala Senior Research Scientist at Singapore Center for 3D Printing Nanyang Technological University Singapore.

\section{Author's Contributions}

All the authors contributed equally to prepare, develop and carry out this manuscript.

\section{Ethics}

This article is original and contains unpublished material. The corresponding author confirms that all of the other authors have read and approved the manuscript and no ethical issues involved.

\section{References}

Annunziata, M., R. Aversa, A. Apicella, A. Annunziata and D. Apicella et al., 2006. In vitro biological response to a light-cured composite when used for cementation of composite inlays. Dental Mater., 22: 1081-1085. DOI: 10.1016/j.dental.2005.08.009 
Apicella, D., R. Aversa, M. Tatullo, M. Simeone and S. Sayed et al., 2015. Direct restoration modalities of fractured central maxillary incisors: A multi-levels validated finite elements analysis with in vivo strain measurements. Dental Mater., 31: e289-e305. DOI: $10.1016 /$ j.dental.2015.09.016

Apicella, D., M. Veltri, P. Balleri, A. Apicella and M. Ferrari, 2011. Influence of abutment material on the fracture strength and failure modes of abutmentfixture assemblies when loaded in a bio-faithful simulation. Clin. Oral Implants Res., 22: 182-188. DOI: $10.1111 /$ j.1600-0501.2010.01979.x

Apicella, D., R. Aversa, E. Ferro, D, Ianniello and A. Apicella, 2010. The importance of cortical bone orthotropicity, maximum stiffness direction and thickness on the reliability of mandible numerical models. J. Biomed. Mater. Res. Part B Applied Biomater., 93: 150-163. DOI: 10.1002/jbm.b.31569

Ashman, R.B., S.C. Cowin, W.C. Van Buskirk and J.C. Rice, 1984. A continuous wave technique for the measurement of the elastic properties of cortical bone. J. Biomechan., 17: 349-361. DOI: 10.1016/0021-9290(84)90029-0

Ashman, R.B. and J.Y. Rho, 1988. Elastic modulus of trabecular bone material. J. Biomechan., 21: 177-81. DOI: 10.1016/0021-9290(88)90167-4

Aversa, R., D. Apicella, L. Perillo, R. Sorrentino and F. Zarone et al., 2009. Non-linear elastic threedimensional finite element analysis on the effect of endocrown material rigidity on alveolar bone remodeling process. Dental Mater., 25: 678-690. DOI: $10.1016 /$ j.dental.2008.10.015

Aversa, R., F.I.T. Petrescu, R.V.V. Petrescu and A. Apicella, 2016. Biomimetic finite element analysis bone modeling for customized hybrid biological prostheses development. Am. J. Applied Sci., 13: 1060-1067. DOI: 10.3844/ofsp.10904

Beaupre, G.S. and W.C. Hayes, 1985. Finite element analysis of a three-dimensional open-celled model for trabecular bone. J. Biomech. Eng., 107: 249-56. DOI: $10.1115 / 1.3138550$

Burnstein, A., D.T. Reilly and M. Martens, 1976. Aging of bone tissue: Mechanical properties. J. Bone Joint Surgery, 58: 82-86. PMID: 1249116

Carter, D.R. and W.C. Hayes, 1977. The compressive behavior of bone as a two-phase porous structure. J. Bone Joint Surgery, 59A: 954-962. PMID: 561786

Dalstyra, M., R. Huiskes, A. Odgaard and L. Van Erning, 1993. Mechanical and textural properties of pelvic trabecular bone. J. Biomechan., 26: 349-361. PMID: 8478354

Gramanzini, M., S. Gargiulo, F. Zarone, R. Megna and A. Apicella et al., 2016. Combined microcomputed tomography, biomechanical and histomorphometric analysis of the peri-implant bone: A pilot study in minipig model. Dental Mater., 32: 794-806.

DOI: $10.1016 /$ j.dental.2016.03.025
Huiskes, R., H. Weinans, H.J. Grootenboer, M. Dalstra and B. Fudula et al., 1987. Adaptive boneremodeling theory applied to prosthetic-design analysis. J. Biomech., 20: 1135-1150. DOI: 10.1016/0021-9290(87)90030-3

Kumar, A., A. Rathi, J. Singh and N.K. Sharma, 2016. Studies on titanium hip joint implants using finite element simulation. Proceedings of the World Congress on Engineering, Jun 29-1, London, U.K., pp: $1-2$.

Kummer, B., 1986. Biomechanische Grundlagen der Statik des Hüftgelenks. Zeitschrift Orthop. Grenzgebiete, 124: 179-187. DOI: $10.1055 / \mathrm{s}-2008-1044544$

Mazaheri, M., K. Hassani, A. Karimi and F. Izadi, 2016. Finite element study of composite materials as an alternative for metal hip prothesis using variable load. Mater. Focus, 5: 430-435.

DOI: $10.1166 /$ mat.2016.1342

Mirsayar, M.M. and P. Park, 2016. Modified maximum tangential stress criterion for fracture behavior of zirconia/veneer interfaces. J. Mechan. Behavior Biomed. Mater., 59: 236-240.

DOI: $10.1016 /$ j.jmbbm.2015.11.037

Mullender, M.G. and R. Huiskes, 1995. Proposal for the regulatory mechanism of Wolff's law. J. Orthop. Res., 13: 503-512. DOI: 10.1002/jor.1100130405

Oh, I. and W.H. Harris, 1976. Proximal strain distribution in the loaded femur. An in vitro comparison of the distributions in the intact femur and after insertion of different hip-replacement femoral components. J. Bone Joint Surgery, 60: 75-85. PMID: 624762

Perillo, L. R. Sorrentino, D. Apicella, A. Quaranta and E. Gherlone et al., 2010. Nonlinear visco-elastic finite element analysis of porcelain veneers: A submodelling approach to strain and stress distributions in adhesive and resin cement. J. Adhesive Dent., 12: 403-413.

Reilly, D.T. and A.H. Burnestain, 1975. The elastic and ultimate properties of compact bone tissue. J. Biomechan., 8: 393-405. DOI: 10.1016/0021-9290(75)90075-5

Reilly, D.T. and A.H. Burstein, 1974. The mechanical properties of cortical bone. J. Bone Joint Surgery, 56: 1001-1021.

Rohlmann, A., U. Mossner, G. Bergmann and R. Kolbel, 1982. Finite-element-analysis and experimental investigation of stresses in a femur. J. Biomed. Eng., 4: 241-246. DOI: 10.1016/0141-5425(82)90009-7

Sorrentino, R., D. Apicella, C. Riccio, E. Gherlone and F. Zarone et al., 2009. Nonlinear visco-elastic finite element analysis of different porcelain veneers configuration. J. Biomed. Mater. Res. Part B Applied Biomater., 91: 727-736.

DOI: 10.1002/jbm.b.31449 
Sorrentino, R., R. Aversa, V. Ferro, T. Auriemma and F. Zarone et al., 2007. Three-dimensional finite element analysis of strain and stress distributions in endodontically treated maxillary central incisors restored with diferent post, core and crown materials. Dent. Mater., 23: 983-993.

DOI: $10.1016 /$ j.dental.2006.08.006

Gottesman, T. and Z. Hashin, 1980. Analysis of viscoelastic behaviour of bones on the basis of microstructure. J. Biomechan., 13: 89-96.

DOI: 10.1016/0021-9290(80)90182-7
Taylor, D., J.G. Hazenberg and T.C. Lee, 2007. Living with cracks: Damage and repair in human bone. Nat. Mater., 6: 263-268. DOI: 10.1038/nmat1866

Weinans, H., R. Huiskes and H.J. Grootenboer, 1992. The behavior of adaptive bone-remodeling simulation models. J. Biomech., 25: 1425-1441. DOI: 10.1016/0021-9290(92)90056-7 